\title{
Re: Preclinical Evaluation of a TEX101 Protein ELISA Test for the Differential Diagnosis of Male Infertility
}

\author{
Korbakis $D^{1,2}$, Schiza $C^{1,3}$, Brinc $D^{3}$, Soosaipillai $A^{3}$, Karakosta $T D^{1,4}$ Légaré $C^{5}$, Sullivan $R^{5}$, Mullen $B^{3}$, Jarvi $K^{2,6}$, Diamandis $E P^{1,2,3,4}$, \\ Drabovich $\mathrm{AP}^{1,3,4}$ \\ ${ }^{1}$ University of Toronto, Department of Laboratory Medicine and Pathobiology, Toronto, Canada \\ ${ }^{2}$ Lunenfeld-Tanenbaum Research Institute, Mount Sinai Hospital, Toronto, Canada \\ ${ }^{3}$ Mount Sinai Hospital, Clinic of Pathology and Laboratory Medicine, Toronto, Canada \\ ${ }^{4}$ University Health Network, Department of Clinical Biochemistry, Toronto, Canada \\ ${ }^{5}$ Centre de Recherche du Centre Hospitalier Universitaire (CHU) de Québec, Université Laval, Faculté de Medicine, Département d'Obstétrique, \\ Gynécologie et Reproduction, Québec, Canada \\ ${ }^{6}$ Mount Sinai Hospital, Department of Surgery, Division of Urology, Toronto, Canada
}

BMC Med 2017;15:60. doi: 10.1186/s12916-017-0817-5.

\section{EDITORIAL COMMENT}

Seminal plasma (SP) is enriched with testis-derived proteins, mRNA and metabolites. Due to blood-testis and blood-epididymis barriers, testis-specific biomarkers are not found in blood serum. Therefore, evaluation of semen and SP could be an option for noninvasive diagnosis of male infertility. In this study, the authors evaluated the performance of TEX101 ELISA in large cohort of fertile, subfertile and infertile men besides the validation of this test as a prognostic biomarker of sperm retrieval in patients with nonobstructive azoospermia (NOA). Impressively, TEX101 differentiated between pre- and post-vasectomy samples with 100\% sensitivity and 100\% specificity. As a marker of differentiation between NOA and obstructive azoospermia, combination of TEX101 with ECM1 increased sensitivity to detect NOA from $69 \%$ to $81 \%$ at 100\% specificity. In addition, a cut-off value of TEX101 $\geq 0.6 \mathrm{ng} / \mathrm{mL}$ provided $73 \%$ sensitivity at $64 \%$ specificity for predicting sperm or spermatid retrieval in men with NOA. The prediction of sperm retrieval by TEX101 was comparable to blood serum protein (the area under the curve value $=0.64 ; 71 \%$ sensitivity at $68 \%$ specificity) (1). Testicular sperm extraction is still the only option for men with NOA. Most of the patients and supposes accept sperm extraction procedure even if they had a very little chance of sperm recovery. Better biomarkers to predict sperm recovery from testicular sperm extraction operation in NOA patients are still required.

\section{References}

1. Ma Y, Chen B, Wang H, Hu K, Huang Y. Prediction of sperm retrieval in men with non-obstructive azoospermia using artificial neural networks: leptin is a good assistant diagnostic marker. Hum Reprod 2011;26:294-298.

Emre Bakırcıoğlu, MD

๑Copyright 2017 by the Association of Urological Surgery / Journal of Urological Surgery published by Galenos Publishing House. 